\title{
High-speed imaging of short wind waves by shape from refraction
}

D. Kiefhaber

\author{
S. Reith \\ R. Rocholz \\ B. Jähne \\ bernd.jaehne@iwr.uni-heidelberg.de
}

\begin{abstract}
Universität Heidelberg, Institut für Umweltphysik, INF 229, 69120 Heidelberg, Germany Universität Heidelberg, Heidelberg Collaboratory for Image Processing (HCI)

Universität Heidelberg, Institut für Umweltphysik, INF 229, 69120 Heidelberg, Germany Universität Heidelberg, Heidelberg Collaboratory for Image Processing (HCI)

Universität Heidelberg, Institut für Umweltphysik, INF 229, 69120 Heidelberg, Germany now at: Heidelberg Engineering, Tiergartenstraße 15, 69121 Heidelberg, Germany

Universität Heidelberg, Institut für Umweltphysik, INF 229, 69120 Heidelberg, Germany Universität Heidelberg, Heidelberg Collaboratory for Image Processing (HCI)
\end{abstract}

This paper introduces the first high-speed system for slope imaging of wind-induced short water waves. The imaging slope gauge method is used, which is based on the shape from refraction principle. The downward looking camera with a telecentric lens observes the refraction of light rays coming from a high power custom telecentric LED light source that is placed underneath the wind wave facility. The light source can be programmed to arbitrary intensity gradients in the $x$ - and $y$-direction, so that the origin of a light ray is coded in intensity. Four gradient images (acquired at $6000 \mathrm{fps}$ ) are combined for one $2 \mathrm{D}$ slope image. By only using intensity ratios, the measurements become independent of lens effects from the curved water surface and inhomogeneities in the light source. Independence of wave height is guaranteed by using telecentric illumination and telecentric imaging. The system is capable to measure the slopes of a wind-driven water surface in the Heidelberg Aeolotron wind-wave facility on a footprint of $200 \times 160 \mathrm{~mm}$ with a spatial resolution of $0.22 \mathrm{~mm}$ and a temporal resolution of more than $1500 \mathrm{fps}$. For the first time, it is now possible to investigate the structure of short wind-induced waves with sufficient spatial and temporal resolution to study their dynamic characteristics without aliasing effects. Example images and a video of a 3D reconstructed water surface are shown to illustrate the principle.

[DOI: http://dx.doi.org/10.2971/jeos.2014.14015]

Keywords: Imaging slope gauge, shape from refraction, wave slope imaging, gravity-capillary waves, air sea interaction

\section{INTRODUCTION}

\subsection{Short ocean waves}

Short wind waves with wavelengths of less than a couple of metres down to a few millimetres are important in many contexts. Surface roughness on this scale determines the strength of radar backscatter [1], the distribution of the slopes of smallscale waves is important for (ocean) radiative transfer modelling [2, 3], they affect remote ocean colour measurements [4]. They also play a prominent role as a major source of near-surface turbulence, influencing the exchange of heat, mass, and momentum between the atmosphere and the ocean [5]-[7].

The measurement of these small-scale waves is challenging: Their amplitudes are orders of magnitude smaller than those of the longer waves, so that all amplitude-based measurement instruments need a very high dynamic range. Surface slope on the other hand has a limited range, since steep waves become unstable and break. In fact, the slopes of very short (capillary) waves, for which the water's surface tension contributes significantly as a restoring force, are even higher than those of longer waves.
A second effect of the surface tension has significant consequences for small-scale wave measurement: the water surface is always smooth at the scale of optical wavelengths. Due to this, all reflections are fully specular. This prohibits the use of standard surface reconstruction techniques (shape from stereo, shape from shading) that have been developed for Lambertian surfaces [8]. Specialized measurement techniques have been developed that use the dependence of the reflection coefficient on the incidence angle of light [9] (known as Stilwell photography in oceanography or deflectometry in industrial inspection [10, 11]). Unfortunately, the reflectance of the water surface is highly non-linear. For incidence angles of $0^{\circ}-40^{\circ}$, it barely changes, above Brewster's angle $\left(53^{\circ}\right)$ it increases rapidly. In addition, very homogeneous (or well known) illumination is required. An extension of the technique that uses the degree and orientation of polarization of reflected light for slope measurements has recently been developed [12]. The dependence of these quantities on the incidence angle is more linear than that of the reflection coefficient. The illumination is not required to be homogeneous, since only the ratio of measured intensities is evaluated, but it needs to be fully unpolarized (or have known polarization). 
For laboratory applications, where mounting parts of the instrument under water is possible, refraction-based measurements are superior to reflection-based techniques for a number of reasons: Only $2 \%$ of the light falling on the water surface are reflected, so that very high luminance light sources are required for reflection measurements. Because the angles are doubled at the point of reflection, these measurements also require a much larger light source. Also, the dependence of total ray deflection on surface slope is less non-linear for refraction measurements [8].

\subsection{Shape from Refraction in Wave Imaging}

A slope meter based on the refraction of light at the water surface was first used by Cox [13]. With a telescope and a photometer he observed light refracted at the water surface. The origin of the light rays could be determined because the light source intensity had a spatial gradient. Unfortunately, in this simple setup the measured intensity was also depending on wave height and surface curvature.

The measurement scheme was inverted in the 1970s, when the laser slope gauge was developed [14]-[16]: The refraction of a vertical laser beam falling down onto the water surface is tracked with an underwater camera observing a screen. Measurements are independent of curvature and, if the screen is placed in the focal plane of a lens, of water height. Still, measurements were limited to single points on the water surface, although attempts were made to scan the laser over an area on the water surface [17].

Real 2D measurements required an extension of Cox's technique to the imaging slope gauge: A camera is looking down onto the water surface, into refracted light coming from an underwater light source with one or two spatial gradients. This way, it is possible to measure surface slope on a two dimensional footprint. Using a camera and an area light source with one or two spatial gradients, it is possible to measure both slope components on a two-dimensional area [18]-[22]. All previous devices could, however, acquire wave slope image sequences at most at video rates with $60 \mathrm{fps}$. The purpose of this paper is to describe the first optical setup for a wave slope imaging instrument with a) telecentric imaging and illumination and b) a computer-controlled four-gradient LED light source, which is bright enough for high-speed imaging with up to $1500 \mathrm{fps}$, so that even capillary waves with the highest frequencies can be sampled without aliasing.

\section{THE HIGH-SPEED IMAGING SLOPE GAUGE}

\subsection{Imaging setup}

Figure 1 is a schematic of the setup that is used at the Heidelberg Aeolotron wind-wave facility. A high-speed camera is observing the water surface from a distance of $4 \mathrm{~m}$. The water body is illuminated from below with a programmable high power LED light source. On its way to the camera, the light is refracted at the surface.

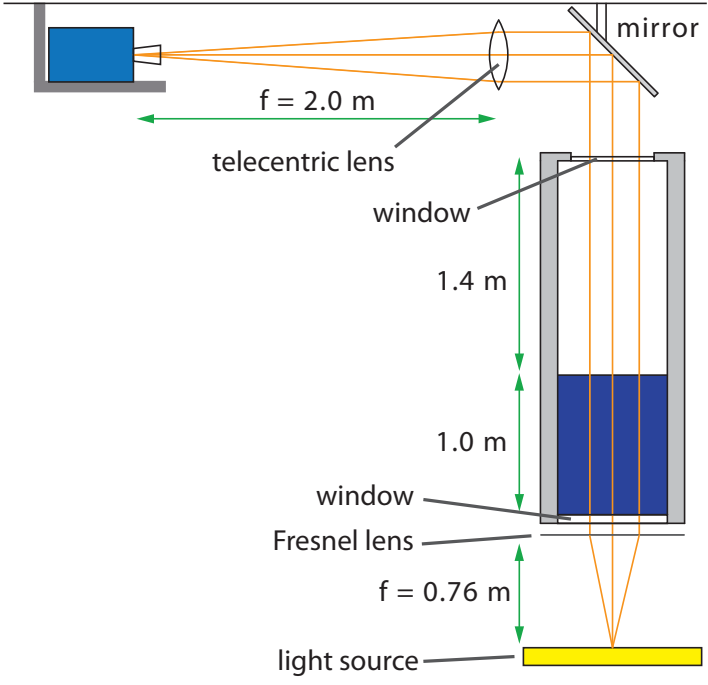

FIG. 1 Wave imaging setup at the Heidelberg circular (annular) wind-wave facility.

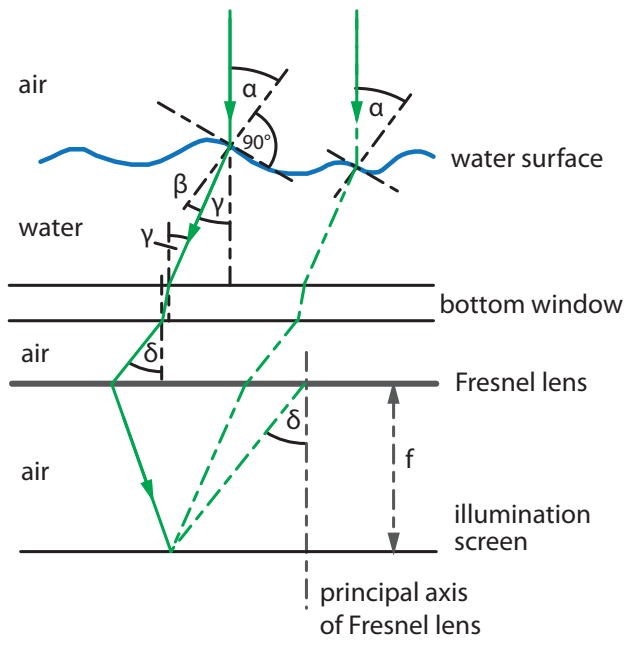

FIG. 2 Ray geometry of the imaging slope gauge.

Both the camera and the water surface are placed in the two focal planes of a large custom BK7 planoconvex lens (diameter $d=0.32 \mathrm{~m}$, focal length $f=2 \mathrm{~m}$ ). Together with a standard $105 \mathrm{~mm}$ camera lens, the two lenses form an object-space telecentric lens, ensuring that all light rays that enter the camera are parallel on the right side of the lens. They are also orthogonal to the mean water surface. As a consequence, the magnification factor is independent of the distance. This is of importance, since wave heights in the Aeolotron facility can exceed $60 \mathrm{~cm}$. At a distance of $4 \mathrm{~m}$, this would lead to changes in the size of structures on the water surface of up to $25 \%$.

The fact that all light rays are orthogonal to the mean water surface is also beneficial for the illumination design. In the absence of diverging rays above the water surface, the light source can be more compact without a loss of measurement range.

Below the water body, an $89 \mathrm{~cm}$ diameter Fresnel lens $(\mathrm{f}=76.2 \mathrm{~cm})$ is used to guarantee telecentric illumination. To understand this, it is helpful to trace a ray from the camera to the light source, as shown in Figure 2: A ray of light penetrating the water vertically from above is refracted at the water surface. For an incidence angle $\alpha$ to the surface normal, the 


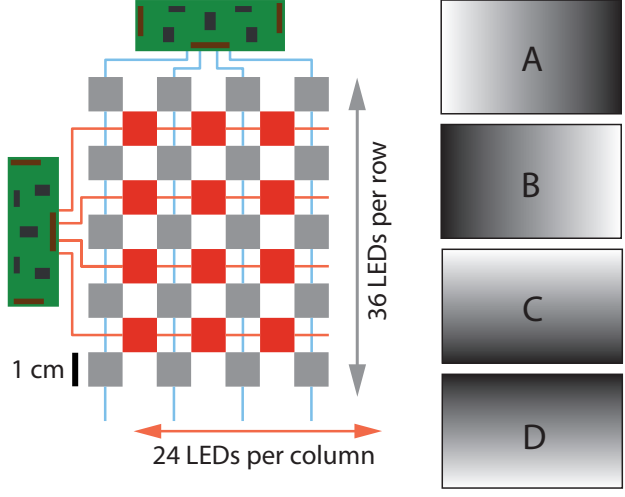

FIG. 3 Sketch of light source; for the $x$ direction, there are 35 columns with 24 LEDs each, for the $y$ direction there are 24 rows with 36 LEDs each. Custom electronics control the LED current of each row/column. Four different brightness gradients (A-D) are pre-programmed.

refracted angle is given by Snell's law $n_{\mathrm{a}} \sin (\alpha)=n_{\mathrm{w}} \sin (\beta)$, where $n_{\mathrm{a}} \approx 1$ and $n_{\mathrm{w}} \approx 1.33$ are the refractive indexes of air and water, respectively. Further refractions occur entering and leaving the polycarbonate bottom window $(n=1.58)$ of the wind-wave facility.

Because the light source is placed in the focal plane of the Fresnel lens, all rays leaving the water at an angle $\delta$ to the vertical are focused onto the same position of the illumination screen. This effectively translates different water surface slopes into unique screen positions, independent of position on the water surface.

\subsection{Position coding}

A light source for the measurement of only one slope component can simply have a gradient in the direction in which slope is to be measured. An extension for two slope components is possible by using a colour-coded light source and an RGB camera [23]. In this scheme, the third colour channel can then be used for normalization and the correction of curvature (lens) effects at the water surface, e.g. by demanding that the total intensity $I=I_{R}+I_{G}+I_{B}$ is constant $[24$, sec. 8.5.3]. Unfortunately, this leads to significant problems with chromatic aberration of the large telecentric lens. In our new setup, a different approach is chosen: The light source consists of columns and rows of LEDs wired in series (see Figure 3). Each row/column can be programmed to two different brightness settings using custom electronics that can control current from $0-1000 \mathrm{~mA}$ with 12 bit resolution and fast multiplexers to swap the two states. In total, the light source consists of 1704 red high power LEDs (Cree XLamp XP-E red, peak wavelength $630 \mathrm{~nm}$, max. cont. current $700 \mathrm{~mA}$ ). The LED spacing is $2.1 \mathrm{~cm}$ in the rows/columns. An acrylic diffusing screen placed at a distance of $4 \mathrm{~cm}$ is used to homogenize the luminance gradient.

With this light source, and the four brightness gradients A - D shown in Figure 3, the following intensity coding scheme can be implemented: Normalized intensities

$$
I_{\mathrm{x}}=\frac{A-B}{A+B} \quad \text { and } \quad I_{\mathrm{y}}=\frac{C-D}{C+D}
$$

in the $\mathrm{x}$ and $\mathrm{y}$ direction are computed from two opposing intensity gradients each. This ratio imaging corrects for lens effects at the water surface, but also significantly reduces the demands to the homogeneity of the light source.

To test the performance and measurement accuracy of the system, a float with a planoconvex BK7 glass lens was put on the water surface (Figure 4(a)). From the known curvature radius of the lens, it is possible to compute the surface slope tan $\alpha^{\prime}$ for every position on the lens. An "equivalent water slope" can be computed from Snell's law (see equations in Figure 4(a)) by setting $\gamma=\gamma^{\prime}$. For small angles, the equivalent water slope is 1.58 times the slope on the BK7 lens.

In Figure 4(b), a raw image of the lens float (illumination gradient $\mathrm{C}$ ) is shown; the axis labels are in units of slope $\left(\tan \alpha^{\prime}\right)$. Even though a diffusing screen is used to homogenize the light source, the individual LEDs can still be distinguished. Also, a number of dents in the bottom window of the facility are visible as darker spots, e.g. at position $s_{x}=0.1$, $s_{y}=-0.25$. In Figure 4(c), the corresponding normalized image (see Eq. 1) is shown. The inhomogeneities have disappeared; a smooth and monotonous gradient corresponding to the slope of the lens in the y direction is visible.

Figure $4(\mathrm{~d})$ shows a profile of the 2D distribution in Figure $4(\mathrm{c})$. The slope in the $\mathrm{y}$-direction is shown as a function of the normalized intensity (which was coded in grey values in Figure 4(c)). The red curve shows the slope of the lens on the calibration float $\left(\tan \alpha^{\prime}\right)$, the blue curve the corresponding water slope that was computed using the considerations in Figure 4(a) $(\tan \alpha)$. The shaded areas indicate the standard deviation of individual normalized images around the mean. The average error of the slope measurement is $0.025^{1}$.

\subsection{High-speed, high-resolution camera}

To avoid aliasing when imaging fast-moving small-scale waves, good resolution is required in both the time and space domain. In our setup, a pco.dimax camera (PCO AG) is used. It allows acquisition of up to $6030 \mathrm{fps}$ at a spatial resolution of $960 \times 768$ pixels. Since four raw images are required to compute a $2 \mathrm{D}$ wave slope image, the effective frame rate for slope measurements is $1507.5 \mathrm{fps}$. The footprint on the water surface is $203 \times 166 \mathrm{~mm}$, corresponding to a pixel size of $0.22 \mathrm{~mm}$. For each image, the integration time is $140 \mu$ s and the LEDs are flashed accordingly. The brightness is sufficient for measurements with f-number 8 (camera lens with $\mathrm{f}=105 \mathrm{~mm}$ ).

\section{RESULTS}

The imaging slope gauge was set up at the Heidelberg Aeolotron wind-wave facility in May 2013. Some processed measurements demonstrating the capabilities of the instrument are shown in the video clip added as supplementary material and represented by Figure 5 .

\footnotetext{
${ }^{1}$ Very recently, the maximum brightness of the light source has been tripled. In future measurements, the error will be smaller.
} 

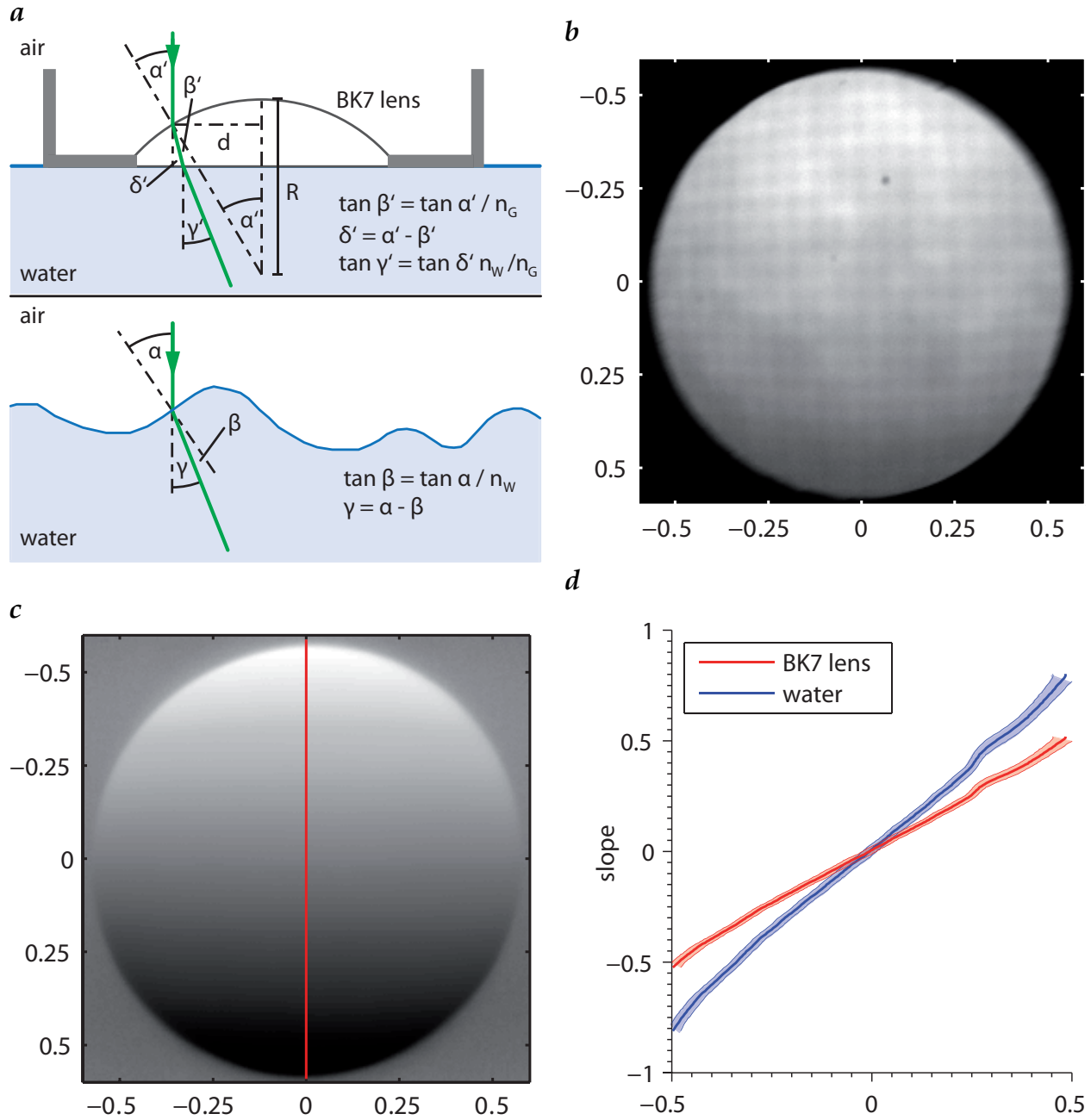

$d$

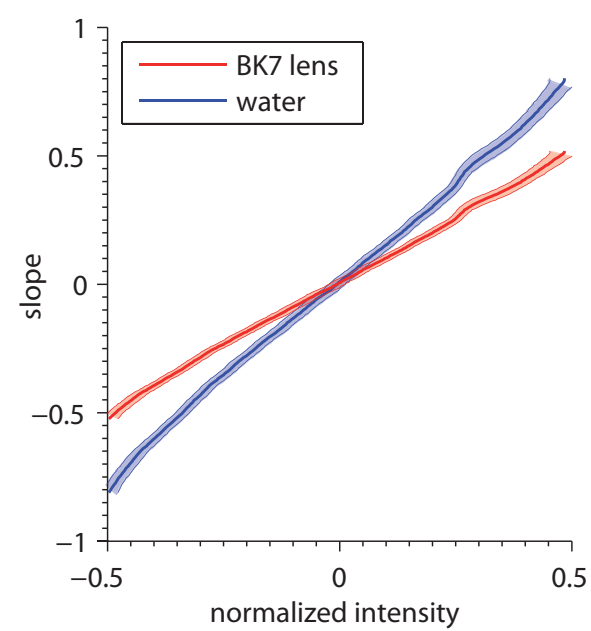

FIG. 4 (a) A float with a BK7 lens is used for validation. The slope $\tan \alpha^{\prime}$ can be converted into the equivalent water slope tan $\alpha$ by applying Snell's law to both scenarios and setting $\gamma^{\prime}=\gamma$. (b) Raw image of a lens floating on the water surface, axes are labelled in units of slope tan $\alpha^{\prime}$. (c) Normalized intensity $I_{\mathrm{y}}$ (see Eq. (1)). (d) The dependence of slope on normalized intensity (red); profile along the red line in c. Also shown is the corresponding equivalent water slope (blue).

The video shows a rendering of the reconstructed 3D surface with software developed by [25]. The necessary conversion from slope to height is done using an algorithm proposed by [26]. The Fourier transform of the height

$$
\hat{h}\left(k_{x}, k_{y}\right)=-\mathrm{i}\left(\frac{k_{x} \hat{s}_{x}+k_{y} \hat{s}_{y}}{k_{x}^{2}+k_{y}^{2}}\right),
$$

with the spatial frequency (wavenumber) $k_{x}, k_{y}$, and the Fourier transforms of the slope images $\hat{s}_{x}, \hat{s}_{y}$. After applying the inverse Fourier transform to the right hand side of Eq. (2), a surface elevation map is obtained. Due to the integration, changes in the mean elevation, e.g. by waves with wavelengths larger than the footprint of the image, are lost. This drawback of wave slope imaging can be made up for by additionally measuring wave height at a single point in the image footprint.

The video shows a typical wave field at medium wind speeds, shown 50 times slower than real time. Characteristic short gravity waves (with wavelengths of about $10 \mathrm{~cm}$ ) are visible, on their leeward side, so-called parasitic capillary waves are formed. These small ripples are created from an instability of the short gravity wave and propagate as bound waves with the same phase velocity as their carrier wave.
In Figure 6, a breaking wave is entering the footprint from the right side. This leads to problems in the reconstruction, since a) multiple refractions can occur when air is entrained in the water or spray is created in the air, and b) surface slopes can become very large, so that the refracted rays miss the light source. In our setup, slopes up to $26.3^{\circ}(\tan \alpha=0.5)$ in the alongwind and $19.6^{\circ}(0.37)$ in the crosswind direction can be measured.

While these $3 \mathrm{D}$ visualizations are a useful tool to observe the dynamics of the wavy water surface, quantitative physical information is typically analysed with the help of 3D wavenumber-frequency power spectra, which contain the distribution of energy to the different wavelengths, as well as the dispersion relation $\omega(k)$. Wavelengths of individual waves can also be extracted with spatial band-pass filtering (e.g. Gabor filters, see [24], sec. 13.4.5).

The complex spatio-temporal evolution of a wind wave field can also be studied with the help of space-time images (Figure 7). Each column of the image contains a slope profile of the water surface, along a line (of length $20 \mathrm{~cm}$ ) in the wind direction. The slope is coded in grey values, white is a high slope away from the wind, black is towards the 


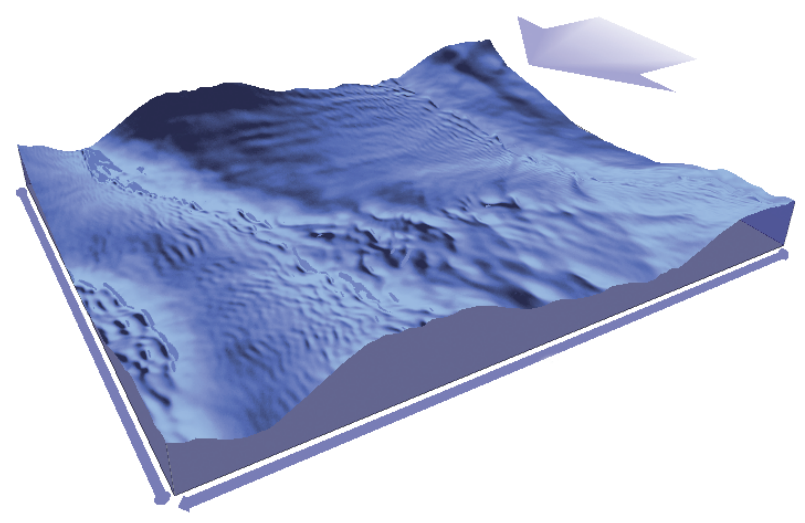

FIC. 53 D rendering of the wave field in the Aeolotron facility at $6.4 \mathrm{~m} / \mathrm{s}$. Characteristic short gravity waves with parasitic capillaries (the small ripples) are visible. Video

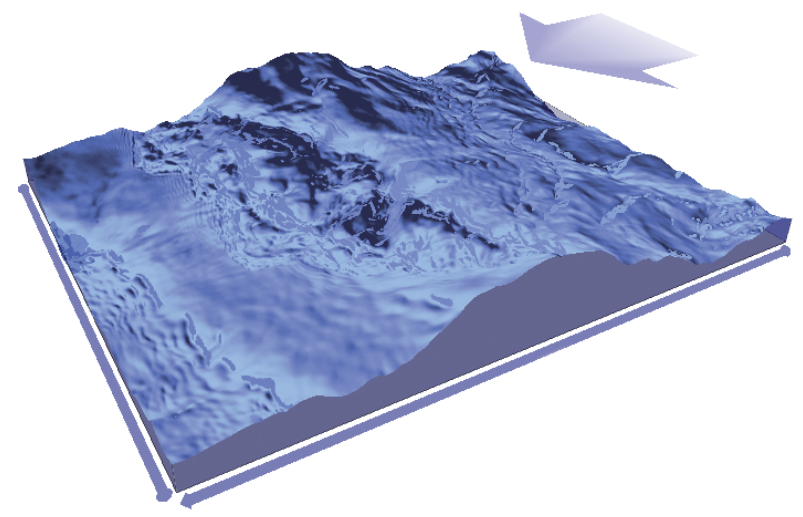

FIG. 6 At higher wind speeds $(8.5 \mathrm{~m} / \mathrm{s})$, breaking waves cause problems: Multiple refraction of the light occurs at bubbles and spray, large slopes outside the measurement range are common.

wind. In the space time image, the wind direction is from top to bottom. The successive columns of the space-time image show the time evolution of the slope profile over a span of $330 \mathrm{~ms}$.

A (microscale) breaking short gravity wave is passing through the ISG footprint (yellow/1). Parasitic capillary waves (very small-scale ripples) that are created on the leeward side are clearly visible. The breaking process is more subtle than with large scale waves; no bubbles are created in the process. After the microbreaker has passed, the water surface is flat, waves are destroyed by the turbulence created in the breaking process. Not all waves travel in the direction of the wind; parallel to the red arrow (2) weak structures are visible that propagate against the wind direction. The modulation of the propagation of smaller waves by the orbital velocity of the dominant larger waves is also visible (blue/3). The blue arrows, which follow the local space-time gradient clearly have different slopes. The local space-time gradient can be extracted by image process-

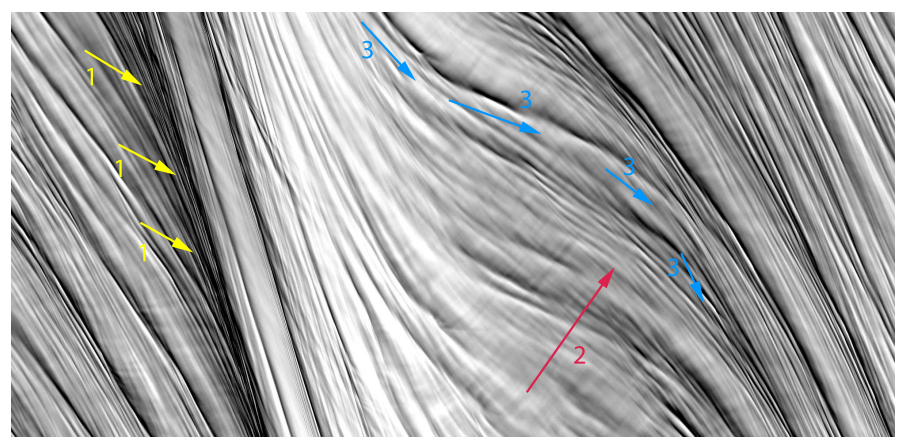

FIG. 7 Space-time image of the wave field at a wind speed of $4.2 \mathrm{~m} / \mathrm{s}$. Each column contains a snapshot of the slope along a $20 \mathrm{~cm}$ transect of the surface. The image shows the temporal evolution of the slope; time increases to the right (330 ms total), the wind is blowing from top to bottom. Slope is coded in grey values, with slopes away from the wind shown in white.

ing to yield the (modulated) velocity of the waves (e.g. [24], sec. 14.2.4).

\section{CONCLUSION AND OUTLOOK}

We have installed the first high speed system for measuring the dynamics of small-scale water waves. The instrument captures the two-dimensional distribution of surface slope with a rate of more than $1500 \mathrm{fps}$ and a spatial resolution of $0.22 \mathrm{~mm}$. With this system, measurements of 3D frequencywavenumber spectra of short waves down to the smallest ripples are possible for the first time without aliasing. With this system, we can study the dynamics and interactions of smallscale waves, e.g. deviations from the linear dispersion relation or non-linear interactions.

At the same footprint on the water surface, we can also visualize the horizontal structure of the mass boundary layer, allowing us to investigate interactions between waves, near-surface turbulence and gas exchange [27].

\section{ACKNOWLEDGEMENTS}

This work is based on a presentation at the "1st EOS Topical Meeting on Frontiers of Optical Imaging" held in Murten $(\mathrm{CH})$ in September 2013. Partial financial support for the project by the German Federal Ministry of Education and Research (BMBF) joint project "Surface Ocean Processes in the Anthropocene" (SOPRAN, FKZ 03F0611F) is gratefully acknowledged.

\section{References}

[1] G. R. Valenzuela, "Theories for the interaction of electromagnetic and ocean waves - A review," Bound.-Lay. Meteorol. 13, 61-85 (1978).

[2] Z. Jin, T. P. Charlock, K. Rutledge, K. Stamnes, and Y. Wang, "Analytical solution of radiative transfer in the coupled atmosphereocean system with a rough surface," Appl. Optics 45, 7443-7455 (2006). 
[3] M. Hieronymi, "Monte Carlo code for the study of the dynamic light field at the wavy atmosphere-ocean interface," J. Europ. 0pt. Soc. Rap. Public. 8, 13039 (2013).

[4] H. R. Gordon and M. Wang, "Retrieval of water-leaving radiance and aerosol optical thickness over the oceans with SeaWiFs: a preliminary algorithm," Appl. Optics 33, 443-452 (1994).

[5] B. Jähne, K. 0. Münnich, R. Bösinger, A. Dutzi, W. Huber, and P. Libner, "On the parameters influencing air-water gas exchange," J. Geophys. Res. 92, 1937-1950 (1987).

[6] N. M. Frew, E. J. Bock, U. Schimpf, T. Hara, H. Haußecker, J. B. Edson, W. R. McGillis, et al., "Air-sea gas transfer: Its dependence on wind stress, small-scale roughness, and surface films," J. Ceo-

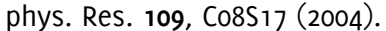

[7] R. Wanninkhof, W. E. Asher, D. T. Ho, C. Sweeney, and W. R. McGillis, "Advances in quantifying air-sea gas exchange and environmental forcing," Annu. Rev. Mar. Sci. 1, 213-244 (2009).

[8] B. Jähne, J. Klinke, and S. Waas, "Imaging of short ocean wind waves: a critical theoretical review," J. Opt. Soc. Am. 11, 2197-2209 (1994).

[9] D. J. Stilwell, "Directional energy spectra of the sea from photographs," J. Geophys. Res. 74, 1974-1986 (1969).

[10] 0. Kafri and I. Glatt, "Moire Deflectometry: A Ray Deflection Approach To Optical Testing," Opt. Eng. 24, 246944-246944- (1985).

[11] M. Knauer, J. Kamisnski, and G. Häusler, "Phase Measuring Deflectometry: a new approach to measure specular free-form surfaces," Proc. SPIE - Optical Metrology in Production Engineering 5457, 366-376 (2004).

[12] C. Zappa, M. Banner, H. Schultz, A. Corrada-Emmanuel, L. Wolff, and J. Yalcin, "Retrieval of short ocean wave slope using polarimetric imaging," Meas. Sci. Technol. 19, 055503 (13pp) (2008).

[13] C. S. Cox, "Measurement of slopes of high-frequency wind waves," J. Mar. Res. 16, 199-225 (1958).

[14] R. Tober, R. Anderson, and 0. Shemdin, "Laser instrument for detecting water ripple slopes," Appl. Optics 12, 788-794 (1973).

[15] J. C. Scott, "An optical probe for measuring water wave slopes," J. Phys. E Sci. Instrum. 7, 747-749 (1974).

[16] B. A. Hughes, R. W. Grant, and R. W. Chappell, "A fast response surface-wave slope meter and measured wind-wave moments," Deep-Sea Res. 24, 1211-1223 (1977).
[17] E. J. Bock and T. Hara, "Optical Measurements of Ripples Using a Scanning Laser Slope Gauge," Proc. SPIE - Optics of the Air-Sea Interface 1749, 272 (1992).

[18] W. C. Keller and B. L. Gotwols, "Two-dimensional Optical Measurement of Wave Slope," Appl. Optics 22, 3476-3491 (1983).

[19] B. Jähne, Transfer processes across the free water interface (Habilitation thesis, Institut für Umweltphysik, Fakultät für Physik und Astronomie, University Heidelberg, 1985).

[20] B. Jähne and K. Riemer, "Two-dimensional wave number spectra of small-scale water surface waves," J. Geophys. Res. 95, 11531-11646 (1990).

[21] X. Zhang and C. S. Cox, "Measuring the two-dimensional structure of a wavy water surface optically: A surface gradient detector," Exp. Fluids 17, 225-237 (1994).

[22] G. Balschbach, J. Klinke, and B. Jähne, "Multichannel shape from shading techniques for moving specular surfaces," in Proceedings of 5th European Conference on Computer Vision (ECCV), 170-184 (Freiburg, 1998).

[23] R. Rocholz, Spatiotemporal Measurement of Short Wind-Driven Water Waves (Dissertation, Institut für Umweltphysik, Fakultät für Physik und Astronomie, University Heidelberg, 2008).

[24] B. Jähne, Digital Image Processing (Sixth Edition, Springer, Berlin, 2005).

[25] S. Wanner, C. Sommer, R. Rocholz, M. Jung, F. A. Hamprecht, and B. Jähne, "A Framework for Interactive Visualization and Classification of Dynamical Processes at the Water Surface," in 16th International Workshop on Vision, Modelling and Visualization, K. P. Peter Eisert, Joachim Hornegger, eds., 199-206 (Eurographics Association, Coslar, 2011).

[26] R. T. Frankot and R. Chellappa, "A Method for Enforcing Integrability in Shape from Shading Algorithms," IEEE T. Pattern Anal. 10, 439-451 (1988).

[27] C. Kräuter, D. Trofimova, D. Kiefhaber, N. Krah, and B. Jähne, “HighResolution 2-D Fluorescence Imaging of the Mass Boundary Layer at Free Water Surfaces," to be published in J. Europ. 0pt. Soc. Rap. Public. (2014). 